\title{
Frontières
}

\section{Frousses, désir, frousse, désirs...}

\section{Luce Des Aulniers}

Volume 12, numéro 2, printemps 2000

Peur bleue...

URI : https://id.erudit.org/iderudit/1074391ar

DOI : https://doi.org/10.7202/1074391ar

Aller au sommaire du numéro

Éditeur(s)

Université du Québec à Montréal

ISSN

1180-3479 (imprimé)

1916-0976 (numérique)

Découvrir la revue

Citer ce document

Des Aulniers, L. (2000). Frousses, désir, frousse, désirs... Frontières, 12(2), 3-9. https://doi.org/10.7202/1074391ar d'utilisation que vous pouvez consulter en ligne.

https://apropos.erudit.org/fr/usagers/politique-dutilisation/ 


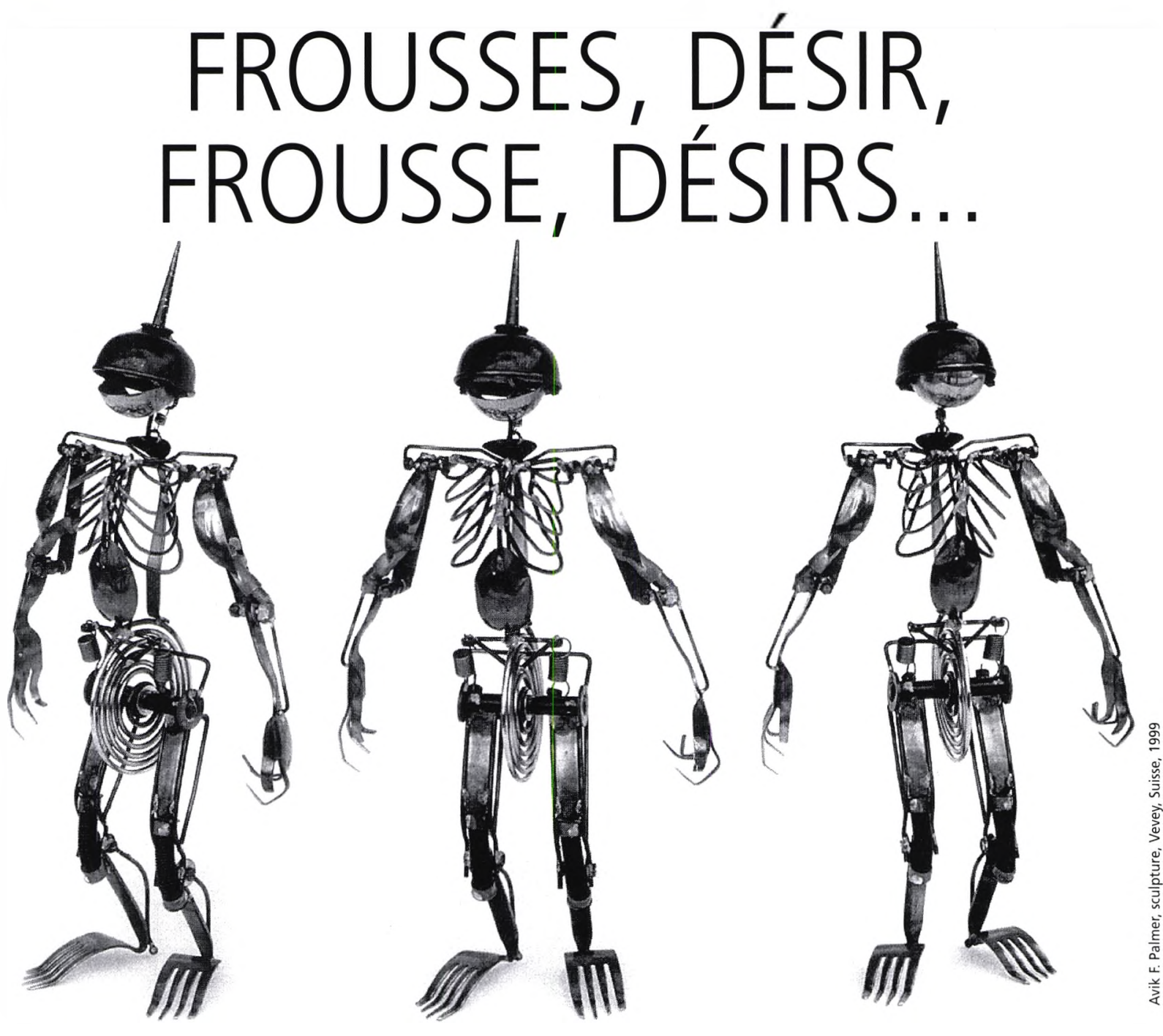

\begin{abstract}
"UN ENFANT M'A DIT: "LA PIERRE EST UNE GRENOUILLE ENDORMIE."
UN AUTRE ENFANT M'A DIT: "LE CIEL, C'EST DE LA SOIE TRĖS FRAGILE." UN TROISIĖME ENFANT M'A DIT: "L'OCÉAN, QUAND ON LUI FAIT PEUR, IL CRIE." JE NE DIS RIEN, JE SOURIS. LE RÊVE DE L'ENFANT, C'EST UNE LOI ET PUIS JE SAIS QUE LA PIERRE, VRAIMENT, EST UNE GRENOUILLE MAIS AU LIEU DE DORMIR, ELLE ME REGARDE.»
\end{abstract}

ET NOUS LA REGARDONS.

Luce Des Aulniers,

anthropologue, professeure au Département des communications et au Centre d'études sur la mort, directrice sortante de la revue Frontières, UQAM.

"La peur mène le monde» ${ }^{2}$. C'est sous cette affirmation, par trop catégorique, en même temps que boutade d'autodérision, que j'avais proposé en 1998 ce thème au comité de rédaction de Frontières.

L'amour mènerait aussi le monde, si on s'en tient aux paroles qui accompagnent l'air. C'est une hypothèse, éternellement à vérifier.
Cette peur serait sans doute l'émotion la plus primitive et en même temps celle qui nous conduit aux plus grands raffinements, qu'il s'agisse de civilisation ou de barbarie. Aussi cette ambivalence de la pensée et de l'acte humain devant ce qui effraie n'a de cesse d'appeler le rapport entre nature et culture. Et cette interrogation serait particulièrement essentielle à déployer, puisque nous vivons, semble-t-il, une époque où la part d'inhumanité en l'humain boursoufle dans l'odieux des extinctions de masse (génocides de tous ordres, carnages, exclusions diverses) ou des affamements du Désir 
(par surexcitation des sens, ou au contraire, par normalisation dépressogène).

La peur viendrait donc moucheter ce que nous entendons par humain, surhumain, inhumain, ahumain et les liens des uns aux autres.

\section{PREMIERS OBJETS}

Parmi ces peurs les mieux distribuées chez anthropos, se trouvent celles des mondes qui ne lui sont pas familiers, lui, le terrien qui arpente son île flottante dans le système solaire: le dessous et le dessus.

Au premier coup d'oeil, le dessous connote la répulsion des gouffres insondables, des souterrains, celle de se terrer. Peut-être celle de se taire? Et de se taire à tout jamais? Nous voilà donc déjà au sens second qui pourrait aussi évoquer la difficulté de plonger dans la vérité des choses, des événements, des êtres et de leurs dynamiques. Dans le noyau primitif, justement, on le dira, veiné entre autres de peur.

Quant à lui, le dessus renvoie d'emblée à l'effroi mitigé d'être dans les airs: la peur de voler, en même temps que la puissante attraction qu'exercent sur nous les volatiles, gracieux, ou technologiquement "parfaits», qui investiguent les confins galactiques ${ }^{3} .$. Au second regard, s'élabore peut-être la résistance à s'envoler, à voler de ses propres ailes, à partager non pas seulement le décollage, mais l'envolée. Et puis la méfiance de ce qui apparaît trop ample, trop fou, trop hors d'ordre?

\section{ORIGINAIRES}

À chaque tremblement engravé au plan biopsychique, se répercuteraient de loin en loin les ramifications inconscientes de deux peurs originaires, celle de l'hominien, celle de son petiot. Chacune, à sa manière, puise dans les peurs anthropomorphiques du dessus et du dessous nichées dans notre mémoire. Comment se font-elles entendre?

Le petit enfant rencontre l'Inconnu, d'autant plus terrorisant comme première épreuve de peur, si cet inédit surgit de l'imprévisibilité, de l'inattendu ${ }^{4}$. Comme l'océan, il crie. Quelque temps après, il pourra crier pour éprouver l'effet de sa voix sur ses parents. Ou encore pour savourer cette frange de plaisir entre la peur excitée et le défi d'en moduler l'expression.

Les premiers groupes humains se sentaient totalement démunis dans la force aléatoire des éléments naturels. Ils criaient. Quelque temps après, ils répercutaient sons, grondements, éclairs, mélodies du vent pour insuffler ardeur devant l'adversité et plaisir modulé, rythmé.

La peur se lit d'abord dans la voix. Et dans la musique?

De ces peurs primitives, arc-boutés à elles, sont nés et se sont complexifiés entre autres une conscience, un besoin fondamental ainsi que plusieurs formes d'intelligence.

\section{UNE CONSCIENCE:}

Procédant de la pulsion de conservation, ou encore "du moi individué, psychique et corporel» ${ }^{5}$, la peur nous met en garde des dangers de mort (à moins que pervertie, elle nous fasse aller à son devant, comme des fantassins aveugles). Dès lors, nous n'avons peur qu'à la condition d'avoir conscience de la teneur de la réalité, et, au-dedans de cette réalité, si nous avons conscience informée de notre existence. Or, procédant de la conscience, la peur vient en retour aviver cette dernière. Nous sommes plus conscients et éventuellement plus sages, si nous tenons compte des déclinaisons en fourchette de la peur, du froissement de sourcil à la poudre d'escampette, en passant par les ruses infinies... Et de ce qui les a provoquées. Car quelqu'un qui n'aurait jamais peur serait plus bête qu'un animal (lequel connaît bien ce phénomène et certains systèmes de signalisation qui y sont liés) ou présenterait à tout le moins un caractère téméraire.

"Sois méfiante, disait Hamlet à Ophélie, la peur est la meilleure garante de la sécurité».

\section{UN BESOIN:}

Aussi le sentiment de vulnérabilité sécrété par la peur, en fait ce qui subsiste de cette dernière une fois l'élément perturbateur passé, suscite le besoin de sécurité. Avec le temps, le souci de protéger son intégrité physique et morale se précise.

C'est dans ce souci que le «je» se développe et qu'il affine sa perception des éléments pouvant entamer l'assiette de cette sécurité. En effet, l'individu grandit de la peur de ne plus l'être...

...Jusqu'au moment où, se confondant, cette peur immunologique rampe sur toutes les autres sources d'inquiétude. Or, cette fragilité de l'identité est d'autant plus troublante qu'elle ne se retrouve pas que chez les individus dont le délire vient désorganiser la personnalité. Elle apparaît aussi comme un des traits structurels de nos vies actuelles, non seulement dans la rapidité, mais dans l'imprévisibilité des changements, si bien qu'une identité en vient paradoxalement à ne se définir que dans cette mouvance ou du moins, prioritairement par elle ${ }^{6}$.

Et là, oubliant les racines, on ratatine. La peur s'est transformée en refus et en stérilité.

\section{DES INTELLIGENCES}

Tout de même, cette émotion-socle nous fait jouer sur le boulier de l'intelligence: au plan microscopique, si, comme on vient de le souligner, l'intelligence nous permet de graduer la dangerosité et d'agir en conséquence, c'est bien la raison intelligente, qui, devant la peur déferlante, fera éviter la panique. En définitive, la pensée sur la peur enrichit le réflexe en face d'elle, comme le décodage de ses silhouettes disparates.

Au plan macroscopique maintenant, on trouve la peur et son train de sentiments dans l'éclosion culturelle: «On est loin de mesurer l'importance jouée par le sentiment d'insécurité dans le développement des sociétés, à travers cette permanente volonté de maîtriser leur rapport à la nature. Exercice de l'intelligence pratico-théorique d'un côté, exacerbation de l'imaginaire magico-religieux de l'autre, les cultures entremêlent ces voies d'accès au dépassement ou à l'apaisement de la peur ${ }^{7}$.»

Abordons d'abord la magie: essentiellement, elle tient dans la manipulation du numineux, de ce qui dépasse l'entendement, du mystère sacralisé. L'être magicien appelle à la fois l'admiration et la distance respectueuse, parce qu'il entretient des liens avec le divin ou le supranaturel, liens privilégiés, c'est-à-dire inaccessibles à la plupart des autres mortels. Accueillant, nommant et maniant la peur, un magicien peut tout autant en causer, dépendamment de l'usage politique - des rapports de pouvoirs - qu'il souhaite voir réalisés, lui et l'institution dont il peut être la courroie de transmission. Le sorcier, le prêtre, le guérisseur, le médecin et les spécialistes de tout acabit détiennent ainsi un statut ambigu: s'ils ne vérifient pas leurs assertions concernant la transformation de cette peur et 
s'ils confisquent pour leur propre sécurité la confiance qu'on leur manifeste, ils obstruent les canaux de sens au profit d'une seule croyance, voire au profit de LA croyance, laquelle vient alors supplanter l'analyse des réalités. On le sait tous néanmoins, leur moralisme est d'autant attirant qu'il est rassurant.

On peut sur cette base interroger autant les phénomènes massifs, spectaculaires, que les distillats des modes: d'une part, que redoute à ce point le tyran pour être devenu tortionnaire? Ou encore, en moins "exotique», qu'estce qui alarme les responsables de la santé publique quand ils tardent à dévoiler les données sur une épidémie toxique ou virale?; d'autre part, qu'ignorons-nous consciencieusement, si fort et si bien tapi, à travers le culte de l'apparence, la course au «m'as-tu-vu»? La peur de ne pas avoir ce qui n'a rien à voir avec l'être, on le sait - rhizome sous les masques de l'agression ostentatoire, de la combine sursécurisante et donc infantilisant l'autre et du «success story» découpé en recettes-sauce-médiatique. Concluons provisoirement sur ce qui lie tous ces "symptômes»: plus on force l'autre et plus on étale une assurance-de-bonheur, plus on aurait peur.

En ce sens les arts - ou plutôt les artistes - peuvent se tenir très près de cette barrière de l'enchantement - et forcément, du désenchantement - dans la mesure où ils utiliseraient leur art pour subjuguer: au sens propre, soumettre les autres à leur moi-sujet.

Il n'en reste pas moins que la création demeure un des «expédients», comme une des ripostes au long cours les plus achevées à la peur, qui est bien, justement, peur de l'inaccomplissement ou peur de joindre en soi une forme de divin ${ }^{8}$. Création de soi, création d'œuvres, créations de lieux pour entraîner l'autre plus loin....

Dès lors, la maîtrise (toujours relative) du rapport à la nature, par la distance critique, permet de sonder la portée de nos inventions, notamment sur la peur et sur l'angoisse.

Mais il y a plus, à savoir notre habileté à contrer le caractère terrorisant de cette nature même, manifeste dans l'intelligence pratico-théorique: cette intelligence technique et scientifique a donné lieu au regroupement des humains dans des huttes rondes - rappel de la première source de sécurité, le sein maternel - à l'origine de la notion de ville 9 . Aujourd'hui, la ville sécrète peurs, angoisses et même boulimie de protection.

De la sorte, nous n'entendons pas tant parler de peur que de sécurité. L'ampleur des diktats et des besoins de confort et de sûreté est éloquent d'une peur qui loge en creux.

Que s'est-il donc passé entre les grandes terreurs et la multiplication des filets de sécurité?

\section{HANTISES ARCHAÏQUES ET INQUIÉTUDES MODERNES: LA PEUR PERVERTIE?}

Peut-être que, entre les Anciens et les (post) Modernes, l'humanité, en s'employant à terrasser certaines peurs, aurait oublié LA peur?

Car les Anciens avaient une vision du monde unifiée et craignaient le courroux des dieux à leur endroit dans le déchaînement d'un de ses éléments, qu'il s'abatte du ciel ou qu'il surgisse des forces telluriques.

Nous avons de notre côté une vision du monde atomisée et nous sommes troublés par la menace qui pèse sur la biosphère. Qui peut faire irruption du dessous ou éclore au-dessus de nos têtes. Car non seulement la nature reste inconquise mais elle "crie» les distorsions subies.
À CONDITION DE GAGNER EN SOLIDARITÉ"

- EDGAR MORIN ${ }^{1}$

On connaît le processus. Accouchant de créatures techniques et scientifiques qui, à raison pour une large part, ont augmenté la réalité et le sentiment de conquête sur des univers jusque là étrangement dévastateurs, l'humain a mieux contrôlé ses peurs des dégâts naturels quoique inégalement: famines, épidémies, etc... En revanche, il semble bien peu maîtriser ses soifs de pouvoir qui, de réponse à la peur originelle, sont devenues sources de terreur: ses créatures, après lui avoir procuré le vertige de l'absolu contrôle, dressent désormais l'épouvantail de sa propre disposition destructrice.

C'est ainsi que «les paniques, les confusions de la population comme des autorités responsables témoignent du désarroi mental de notre époque qui refuse un monde cassé, un monde fêlé. Pourquoi? Parce que aujourd'hui, l'homme moderne, nous-mêmes, avons transféré au monde les perfections jadis attribuées au ciel: "la principale occupation de la philosophie consiste à résoudre le problème de l'existence du monde", notait Schelling au début du XIXe siècle. Le sida fait lever la possibilité de la non-existence du monde - comme la bombe atomique aussi inquiétante pour nous qu'était impie autrefois celle de la non-existence de Dieu. ${ }^{10}$ ».

Comment peut-on dès lors relier cette soif de pouvoir et la production de toutes pièces d'un monde qui se dénie lui-même et risque de s'abolir?

«L'acharnement des hommes à conquérir une parcelle de pouvoir (financier, politique...) ne s'explique pas tout uniment par le souci du confort, ou la fascination du prestige, mais plutôt par le besoin impérieux d'accumuler la vie et donc de l'éloigner de la mort. ${ }^{11}$,

Le paradoxe est renversant: la volonté de survie, au sens accumulatoire, aurait amplifié la peur de la mort fondatrice. Cela se passe comme si le propre de toute civilisation, à savoir la «lutte contre le pouvoir dissolvant de la mort» (L.-V. Thomas) se trouvait lui-même débordé, gauchi, perverti: combattre la part de sabotage de toute mort en ne considérant que cette dernière et en se donnant sur le mode de la réaction une surdose de mort. De la sorte, la peur de la mort et la peur tout court seraient devenues inenvisageables, puisque de les ressentir remettrait en question la logique qui imbibe nos vies: on aurait ainsi plus peur de perdre de factices avantages que peur d'avouer notre tremblement fondamental, composante structurante de notre être...

Pourtant, «les échafaudages de secours, diversions ou satisfactions substitutives qu'offre [cette civilisation d'accumulation des biens] pour aplatir l'angoisse de mort sont fortement ébranlés. ${ }^{12} \%$. Ainsi, l'apprenti-sorcier peut à l'occasion, sentir le vertige du vide tonner comme une déflagration de masse, on vient de le souligner. Mais il reste aux prises avec une complexité supplémentaire, celle liée au statut social de la peur.

Car tout à côté de ces épanchements collectifs soudains, la peur rôde à bas-bruit et d'autant qu'on veuille faire assoupir la violence fondatrice de la mort. La peur est de cette manière généralement intériorisée. L'ultra individualisme devient ainsi son abri de prédilection. Une 
nouvelle figure de la peur collective voit le jour, celle qui étreint, sans qu'un mot à son propos ne soit accordé, ou quand c'est le cas, raillé par un cynisme conquérant qui continue sur sa lancée: dénier le tressage vie-mort en inventant toutes sortes d'hybridations, de laboratoire ou de fiction, des graines de tournesol broyées avec un gêne de tortue ou de femmes-machines. Multiplier les formes de l'inquiétant détourne de la peur toute crue.

Car avoir peur fait honte ${ }^{13}$.

Ces vieilles hantises-hontes du dessous et du dessus, souvent ramifiées en angoisse, au bout du compte, se rejoignent dans la peur d'affronter. Car si on entend volontiers le slogan "peur de la peur» ou "peur d'avoir peur», il se peut tout autant que cet énoncé soit lesté d'un difficilement formulable: la peur du courage. Celui-ci tient essentiellement en la capacité de faire l'effort pour discerner et affronter les résidus de l'impensé accumulatoire, voire du paradoxe qui, pour gagner du pouvoir sur la mort, suscite encore plus de morts, visibles et invisibles: l'indifférence, l'intolérance, la bêtise, la veulerie, la duperie qui arborent partout leurs beaux atours, et qui, bénéficiant en bonne partie d'impunité, rongent la confiance sociale. Avec ces corollaires: le ressentiment perçu comme un droit, la jalousie comme mode d'être, et bien sûr, amplifié, le diffus sentiment d'insécurité propre à la post-modernité. Comme l'écrivait encore Louis-Vincent Thomas, "la spirale est infernale ${ }^{14}{ }^{\prime}$. Nous avons dès lors de moins en moins l'impression de participer à la Vie.

On ne s'étonne alors pas que cette insécurité flottante reflue avec violence entre autres autour des mourants: on liquiderait volontiers sa propre sédimentation de tracs quand on "ose» demander: "Avez-vous peur?", comme si cette peur cristallisait toutes les émotions relatives à la mort, comme si cette peur n'avait pas tant d'objets, et comme si, à chaque source de peur, ne pouvaient pas correspondre autant de sources d'espoir. On ne s'étonnera pas non plus de l'aversion d'une mort lente, la rendant systématiquement totalement synonyme d'une dégradation de facultés, dégradation si ahurissante pour nos sensibilités bien rompues au rutilant.

On ne s'étonnera pas plus des difficultés de déchiffrement des peurs de dormir et des peurs de la noirceur, si on ne les interprète pas comme deux grands archétypes de la peur de la mort, tout au long de la vie. Mort symbolique, par l'abandon juste, nécessaire à toute prise et reprise, et préalable à toute ouverture ultérieure. Et bien sûr mort destinale...

\section{LE TEMPS ET SA SOEUR LA MORT}

Avec la peur, la conscience s'étire donc dans un rapport au temps qui fait anticiper: qu'est-ce qu'anticiper si ce n'est projeter nos avants (autrefois, jadis, naguère) plus ou moins digérés dans le futur?

Si d'emblée, les peurs ne sont pas éprouvées et représentées en lien direct avec la mort, tout ce qui précède dans cet éditorial tend à indiquer que la réalité de la mort, rencontrée, fabulée, anticipée, non seulement fonde les peurs, mais en teinte les modes de défense, au point, on l'a dit, de rendre ces derniers mortifères.

«La» mort. Et pourtant, de nos jours les personnifications nord-américaines de la mort la rendent majoritairement masculine, autant de la part des femmes que des hommes $^{15}$. Si, comme le soulignait Philippe Ariès ${ }^{16}$, ces personnifications tiennent lieu de canaux d'expression des orientations émotives et cognitives devant la vie et la mort, il y aurait lieu d'investiguer d'une part le caractère vitalisant et mortiferre des rapports entre les genres et d'autre part, le type d'images proposées dans une production visuelle exponentielle. Ils détermineraient en partie la peur, de la même façon qu'ils seraient déterminés par elle.

Nous n'avons pourtant pas voulu accorder large domaine à la peur de la mort comme événement dans cette livraison de Frontières: bien sûr, les rubriques pourraient s'étendre: peurs de la maladie (et maladie comme réponse à l'anxiété chronique), des accidents et peurs des figures de mort que sont ces maladies. Nous nous sommes plus centrés sur d'autres figures: la perte, la solitude, la folie, l'inconnu, l'absence de recours, la limite... Bien plus, on peut articuler de maintes façons les formes de peurs relatives à l'avènement de la mort: peurs associées au processus de mort, à savoir la douleur, l'indignité et le fait de devenir un fardeau pour l'entourage; peurs liées au sort post mortem concernant le corps, un principe spirituel, l'inconnu au jugement; enfin, peurs liées à l'impossibilité de terminer un projet, que nous avons évoquée plus haut, ou encore à la déperdition des relations...

Ayant toutefois pris connaissance d'une centaine d'articles sur la peur de la mort, nous pouvons sérier ainsi la teneur de nos premiers constats ${ }^{18}$ :

1) Se retrouve en cette matière la même tendance dominante que nous observions en suicidologie, mine de rien, et ce, sous deux aspects: le premier tient aux impensés, si ce n'est aux tabous: la suicidologie ne parle pas ou si peu, de la mort; l'«anxiologie de la mort» (voir plus bas) évoque bien peu le caractère fondateur et dynamique de la peur de la mort et de la peur. Quant au second aspect, on part de la clinicisation, ici, de la peur ${ }^{19}$, on poursuit, pour beaucoup, dans la confusion avec l'anxiété (voir la Présentation pour les distinguos) et on déborde vers la pathologisation sociale. On pourrait y déceler un procédé de pasteurisation javellisante du microbe de la peur. Ainsi nous pourrions déduire que la peur de la mort est d'ordinaire mal prisée dans les milieux de recherche, comme si on y craignait trop et implicitement les ratés et les incertitudes.

2) Naît ainsi une sorte de portrait-robot (même pas idéal type), forcément caricatural (on nous le permettra puisque l'on parle de peur): ne craint la peur et n'a pas peur de la mort l'homme (surtout au masculin) heureux. Il a confiance en lui, en sa capacité de maîtrise du monde (locus of control), il est intégré dans des réseaux, qui religieux, qui «in». Il s'adapte au changement. Bien plus, il le crée. Au contraire, la peur de la mort serait le lot des faibles, des mésadaptés, des «loosers» et bien plus, de ceux qui la perçoivent comme non naturelle, absurde, froide 20.

3) Il n'y a pas ou peu de recherche sur la peur de ce qui cause la mort, et inversement, sur les modèles de «belle mort» ou encore, plus précisément, sur l'aisance à disposer de la vie des autres, qui, elle, est à craindre: on n'a qu'à constater les politiques sur le port d'armes.

4) On ne relie pas plus la peur de la mort aux autres peurs, cherchant à voir comment la première s'y dissémine. La peur de la mort est ainsi dépolitisée, désorganisée, déstructurée.

5) Entre toutes ces circonstances de mort, pas non plus de recherche sur les inégalités sociales, entre pays ou entre groupes de travail ou sur "l'apeurement» que peuvent susciter lesdites inégalités. 
6) Avec l'échelle de Templer (Death Anxiety Scale) ${ }^{21}$, comme dogme scientifique, émerge une conception de la recherche (visible sous d'autres thématiques) qui peut faire frémir tout penchant à l'exploration fondamentale. Bien sûr, la quête des corrélations satisfait notre curiosité. Toutefois, elle ne nourrit que le besoin de description, pas l'impérieux et urgent - si on regarde les regards - désir de comprendre.

7) Il est évident que les troubles de détraquages de la peur, dans l'anxiété, dans l'angoisse, peuvent être traités. Pas dans la condescendance des gagnants et de ceux qui sont passés maîtres dans l'art et du subterfuge et du nivellement.

Bref, la peur de la mort, comme cheville ouvrière de la conscience, serait trop souvent déboulonnée. Ce faisant, participerait-on au déni, non pas tant de la mort, que de la peur, comme impulsion de la réalisation et du désir?

Car lorsqu'on l'appréhende de manière transculturelle, on s'aperçoit bien que la peur est universelle, non seulement du fait qu'elle est partagée par l'humanité, mais également en ceci qu'elle imbibe un tant soit peu l'ensemble de nos choix d'êtres libres. Ou du moins prétendant l'être. Car on le sait, la liberté n'équivaut pas à l'affranchissement de toute dépendance

\section{À QUOI RÊVENT LES PEURS? OU: À CHAQUE PEUR SON DÉSIR}

Sautons maintenant dans le vide, doublé d'un noir bleuté étrange, à éclairer avec la balancelle du désir.

Car avec la capacité de composer avec la peur, vient la capacité de composer avec son désir et avec les limitations de ce désir. On le sait, cela s'est mis à l'épreuve très tôt, dans le conflit intérieur vécu par l'enfant: d'un côté certains désirs, de l'autre, ce qui entre en désaccord avec eux, à savoir sa conscience morale (son Surmoi) et son besoin de préserver une bonne image de lui-même (son Idéal du Moi). C'est de la résolution de ce conflit intérieur que l'enfant sera plus préparé à la fois à surmonter les limitations que la vie impose et à supporter le conflit, la séparation, les situations sources de peur, sans se sentir trop fragilisé en face des autres qu'il verrait tout puissants.

Cette résolution passe par l'admission des limites imposées par la filiation, le sexe et le genre, et bien sûr par notre nature mortelle.

Or, surmonter les limites ne peut s'effectuer sans prendre la peur à bras-le-corps. C'est sur ce corps-là que notre civilisation défaille. Et aussi, forcément, sur ce avec quoi vient la peur. Car admettre la peur fait admettre le désir. Admettre le désir fait admettre la peur que crée le désir. Et toujours, le désir qui sourd de la peur.

Alors «la peur surmontée se traduit par l'éveil du désir, d'un désir dont on méconnaît totalement les limites. De plus, il faut assumer aussi l'agressivité accompagnant toute conquête, d'où l'instauration d'épreuves consubstantielles à tout périple, à toute évolution. Les épreuves justifient alors cette peur de l'autre qui est en réalité peur de soi, comme le chante clairement Aît Menguellet:

Toi qui as coutume de marcher la nuit

Qu'as-tu à craindre du jour?

Hors les ténèbres et le froid

Quel monstre peux-tu rencontrer?

Prends un miroir tu y verras

L'image de celui que tu fuis

Car c'est toi-même que tu crains de rencontrer.»
Car l'autre n'est qu'un support matérialisant ce qui est inscrit dans nos esprits 22 .

Pour assimiler la peur, accepter de risquer pour son désir. Accepter son désir. Et la peur. Alors, s'élançant du désir, nous mourons à nos peurs. Et ces désirs à l'œuvre font éclore d'autres peurs. Frousses, désir, frousse, désirs... le cycle s'avère vital.

Nous espérons que dans cette parution qui clôt aussi un cycle de Frontières, nous regardons une fin dans le sens du dépassement.

Une grenouille, oui, qui devient pierre, qui devient accomplissement d'un secret: laisser à nos lecteurs le soin de se (re)trouver à travers le miroir... et les fourchettes que nous leur tendons.

Les mythes et les fables-récits de transmutation du minéral au végétal, du végétal à l'animal, de l'animal à l'humain (et vice versa) crient et soupirent cette tension dans le dépassement.

Encore une fois, peurs et désirs entrelacés.

\section{Notes}

1 Edgar MORIN, «Les anti-peurs», Communications, no 57, "Peurs», Paris, Seuil, 1993, 193 pages, p. 131-140: 137.

2 La remarque n'est certes pas originale. Pour ce qui concerne de plus près Frontières, soulignons que la peur de la mort comme levier de civilisation a été développée notamment chez nos contemporains, par Edgar MORIN, L'homme et la mort, Paris, Seuil 1971 (1950), par Louis-Vincent THOMAS (dans ses 30 ouvrages, dont ceux cités plus bas) et dans une autre perspective, par Ernest BECKER, dans The Denial of Death, New York, The Free Press, 1973

Le travail de ce dernier est soutenu par le postulat que la peur de la mort tient lieu de principe moteur du comportement humain. Par conséquent, la société est une fiction créée par les individus et source de sens: l'homme se voit lui-même comme un héros de cette fiction, luttant pour fabriquer une liberté qu'il sait fragile, puisqu'il pressent que ses créations meurent avec lui. L'individu construit donc son existence en cherchant une forme d'immortalité (ou en "déniant»sa fragilité) qui allège sa peur de la mort, en attribuant une nature supernaturelle à la culture: cette dernière lui offre les mécanismes de perpétuation et de rédemption. Elle est le véhicule de son désir d'immortalité, lequel sous-tend son appétit de pouvoir: en ce sens, les tenants de pouvoirs héroïques cristallisent sur eux notre désir d'immortalité. Paradoxalement. l'individu peut y parvenir au prix de la vie des autres: le mal consiste donc en un manque de critique des fictions sociales qu'il crée pour réprimer son anxiété et en définitive, à ce besoin tragique d'être victorieux de la mort. En revanche, cette quête de sens ne trouve sa plénitude que dans le sentiment religieux: le sacré, à un niveau de pouvoir invisible et inconnu, référant à Dieu, source de la création. Alors la peur de la mort devient peur d'une mort - et d'une vie - sans signification.

Ajoutons ceci: depuis les trente dernières années, les sciences sociales sont marquées par le courant phénoménologique, tant en anthropologie qu'en psychologie, visible notamment par la recherche sur les significations des pratiques, sentiments, représentations. En ce sens, par ce biais, même si elle l'ignore, la recherche serait imbibée des mécanismes, nous ne dirions pas de déni, mais de négation du pouvoir annihilant de la mort, intégrant le dépassement (toujours relatif) de cette dernière par la quête de sens.

3 Sans oublier l'aéroanxiété, qui resurgit à chaque écrasement d'avion et qui fait souffrir particulièrement les personnes obligées à prendre l'air, comme les gens d'affaires: une situation de bris mécanique dans un gros-porteur, par exemple, est particulièrement anxiogène, dans la mesure où les mécanismes de fuite et d'affrontement actif deviennent inopérants. 
4 Chez les nouveau-nés, apparaissent trois peurs: celle de tomber, celle, justement, de ce qui arrive brusquement, et aussi celle de perdre leur peau protectrice, la dyade qu'ils forment avec la mère. (H. BRUNSCHWIG, "Les peurs enfantines", Soins gynécologiques, Obstétrique, Puériculture, Pédiatrie, no 108, mai 1990, p. 41-44.)

5 Francis PASCHE, "Peur de la mort, angoisse de mort, défense du Moi", Revue Française de Psychanalyse, jan.-mar. 1996, vol. 60, no 1, p. 49-53: 49.

6 Sommes-nous par là éloignés du concept d'identité de l'ego théorisé par Erik E. ERIKSON (notamment: avec H. P. NEWTON, In Search of Commun Ground, New York, W.W. Norton, 1973), dans lequel un des buts de l'identité est précisément de préserver l'individu du sentiment de discontinuité? Bien plus, dans quelle mesure cette définition actuelle majoritaire n'emprunte-t-elle pas aux philosophies orientales, en retenant d'elles non pas tant une expansion de l'ego dans une perspective physicopsychosociale englobée dans la dimension cosmique, mais une expansion de l'ego dans le sens matériel du terme, par accumulation d'expériences individuées sur la durée de l'existence («qui peuvent toujours servir dans un $C V$ », entend-t-on)? Si c'est le cas, comment se porte et se manifeste la peur de la mort-cessation-de-cette accumulation?

7 Bernard PAILLARD, "Appréhender les peurs", responsable du numéro de Communications (voir note 1), auquel nous devons plusieurs idées énergisantes sur le phénomène..., p. 7 16: 12 .

8 Annick DE SOUZENELLE rend compte de cette idée de l'art à la fois comme cri de l'oppression et comme libération liante: "L'image divine est profondément refoulée [en l'Homme], recouverte d'un masque de plomb, mais non altérée. Le refoulement est cependant si profond qu'un abîme sépare désormais l'humain du divin. C'est le franchissement de cet abîme qui fait l'objet de l'insondable inquiétude de l'humanité. Celle-ci l'exprime à l'état pur dans son art, ses chants, sa littérature sacrée comme son folklore. Elle le projette au plan psychique et l'exprime à ce niveau en déferlement confus et nostalgique, vite désorganisé, cédant alors le pas à une marée démoniaque qu'une médecine agnostique ne peut endiguer La nature ontologique de l'Homme est mariage." (soulignés de LDA)

Le symbolisme du corps humain, St-Jean-de-Brayes, Éditions Dangles, 1984, p. 95.

9 Voir à ce propos Yvonne KNIBIEHLER (dir. publ.). Marseillaises: les femmes et la ville, des origines à nos jours, Paris, Côté-Femme, 1993, et M. STONE, Quand Dieu était femme, Montréal, L'Étincelle, 1979 (1976). On trouve ici une hypothèse complémentaire à celle de W. N. RABY, dans le présent numéro.

10 Madeleine PRÉCLAIRE, “Le responsable, c'est toujours l'autre», L'AGORA, été 1995 , p. 35-38, 2e partie de l'article "Les grandes peurs en Occident, mythes ou réalité?", ibid juillet 1995, p. 36-39.

11 Louis-Vincent THOMAS, Mort et pouvoir, Paris, Payot, 1978, p. 134

12 Louis-Vincent THOMAS, Civilisations et divagations, Payot, 1979, p. 44

13 Évidemment, cette assertion peut être nuancée. À titre d'exemple, la peur revient en leitmotivs dans la chanson "Vaste est la vie» (musique de Marie BERNARD, paroles de Michel RIVARD), entonnée par 2300 choristes dans le cadre du concert 2000 voix chantent le monde, à Québec et à Montréal, juin 2000. (Concert impossible à endisquer)

14 Louis-Vincent THOMAS, Mort et pouvoir, ibid. note 11, p. 201. La liste des reflux de ces peurs sous forme de violence aux abords de la mort serait longue: l'euthanasie comme le suicide peuvent en faire partie.

15 R. KASTENBAUM et R. AISENBERG, The Psychology of Death, New York, Springer, 1972;

R. LONETTO, "Personnifications of Death and Death Anxiety", Journal of Personality Assessment, 1982, vol. 46, no 4, p. 404-408;
R. T. MACDONALD, W.A. HILGENDORF, «Death Imagery and Death Anxiety", Journal of Clinical Psychology, jan. 1986 , vol. 42 , no 1 , p. 87-91.

16 Philippe ARIÈS, Images de l'homme devant la mort, Paris, Seuil, 1983.

17 Les dites peurs peuvent être allégées ou exacerbées par la manière dont on entre en relation avec les mourants, notamment atténuées en les aidant à se remémorer leurs manières passées d'en découdre avec la peur... À ce propos, la littérature sur l'individualisme et de manière plus pointue, sur les baby boomers, s'avère éclairante. Entre autres: R. NEALE, "Individualism and Our Fears of Death", Death Education, 1987, vol. 7, p. $25-31$

18 Ce corpus documentaire n'a aucune prétention scientifique (articles écrits entre 1975 et 1996, provenant de périodiques spécialisés (par ordre) en psychologie, en éducation à la mort et en sciences sociales) et a été lu à titre indicatif.

19 Michel FOUCAULT, dans l'au-delà, doit se lamenter sur son immortalité: que fait-on, surtout du côté américain qui pourtant l'avait accueilli, de son (ses) analyses(s), notamment Naissance de la clinique: une archéologie du savoir médical, Paris, PUF, 1988 (1963), regard clinique importé par la psychologie?

20 Pour ce qui concerne la présence plus ou moins différenciée de la peur de la mort, dans une approche interculturelle, deux facteurs liés à la tension individu/collectif semblent au premier regard s'annuler mutuellement: facteur 1) si on se fie à l'approche existentielle de I.D. YALOM (Existential Psychotherapy, New York, Basic Books, 1980), plus un individu se sent en contrôle de son environnement, moins il manifeste d'anxiété et de dépression face à la mort: ce sentiment de contrôle expliquerait le fait que les femmes manifesteraient plus d'anxiété que les hommes en regard de la mort (incidemment, on peut nuancer cette généralité notamment du fait que l'échelle de Templer, largement utilisée, met plus en évidence les aspects émotifs que les aspects cognitifs face à la mort, lesquels, à la base, distinguent les femmes des hommes. L'échelle introduit donc un biais. Voir l'article de, L. ST-AMOUR et M. C. KIELY dans le présent numéro; et J.F.SCHUMAKER, R.A. BARRACLOUGH, et L.M.VAGG, "Death Anxiety in Malaysian and Australian University Students", The Journal of Social Psychology, 1988, vol. 128 no 1, p. 41-47.) (W. REIMER, D.I. TEMPLER, "Death Anxiety, Death Depression, Death Distress, and DeathDiscomfort Differential: Adolescent-Parental Correlations in Filipino and Americans Populations", Omega, vol. 32, no 4, 1995-1996, p. 319-330)

Donc, face au primat du contrôle par l'individu, se pose ceci: facteur 2) une société où les individus sont perçus comme devant coopérer et se coordonner dans l'action et l'attitude en vue du bien commun offre un support social qui allège l'anxiété de la mort. (J.F. SCHUMAKER, W.G. WARREN, G.GROTH-MARNAT, "Death Anxiety in Japan and Australia", The Journal of Social Psychology, 1991, vol. 131, no 4, p. 511-518). Ceci pourrait expliquer comment, face à l'incontrôlable naturel, par exemple, la solidarité est un mode de défense efficace contre l'effroi. Voir intra, G. DUBY.

Proposition théorique de synthèse: Cette peur serait donc en partie allégée par un équilibre entre d'une part l'autonomie individuelle (la capacité de penser par soi-même) et d'autre part le fait de se savoir participant d'une logique sociale, incluant les conflits... Il reste à trouver une culture où un tel équilibre existe.

21 Voir l'article L. ST-AMOUR et M. C. KIELY dans le présent numéro.

22 Tassadit YACINE, "La féminité ou la représentation de la peur dans l'imaginaire social kabyle», Cahiers de littérature orale, no 34, "Le pouvoir de la femme», Paris, Publications Langues'O, 1993, p. 19-46: 29. 


\section{$\begin{array}{lllllllllllllllllllllll} & \text { A } & \text { I } & S & A & U & X & L & E & C & T & E & U & R & S\end{array}$}

Après 3 ans, je signe mon dernier (et long, je l'avoue) éditorial à titre de directrice de Frontières. J'inscris cet avis au «je», contrairement à ma pratique habituelle. Car des raisons d'équilibre physique, mental, intellectuel, m'ont incitée à ne pas demander de renouvellement de mandat, ni à la direction, ni à la rédaction, et ce, en dépit de la joie que me procure ce travail: à la direction, joies de l'action, des découvertes et des mises en relation; à la rédaction, joie de faire émerger l'idée juste et de la rendre, avec ses auteurs. Je n'aurais pas signalé ce départ, n'eût été de l'état de fait qui a présidé à ce choix et qui surtout, ébranle aujourd'hui l'existence de Frontières. Le lecteur doit être mis au courant. Par un hasard d'édition, il trouvera en Hors thème un bilan global à propos duquel j'ajoute ou je précise ici quelques points.

Depuis plusieurs années, la revue se heurte à une indigence de ressources professorales au sein de ses comités et surtout, dans les activités d'exécution. Idéalement, et ce fut le cas lors de la naissance de la revue, en 1988, un professeur assume la direction, un autre, la rédaction et une petite équipe se répartit les responsabilités. C'est à cette condition expresse d'implication professorale qu'une revue acquiert un statut institutionnel et peut bénéficier d'un financement d'organismes externes. Elle développe aussi un indispensable rayonnement.

Or, les deux postes de rédaction et de direction requièrent un type d'engagement que nos collègues de l'Université du Québec à Montréal et autres ne veulent ni ne peuvent manifestement réellement assumer. Les demandes effectuées à l'endroit des départements et des professeurs se sont trouvées sans écho, de même que la formalisation des liens avec nos collaborateurs d'autres universités.

Il y a à cette situation plusieurs motifs qui relèvent notamment de la pénurie de ressources compétentes en études sur la mort ainsi que des objets de recherche de nos collègues, n'attribuant pas aux questions de la mort une place prédominante. Pourquoi? La question est légitime et demanderait une réponse élaborée que je me contenterai de ramener aux retraites de plusieurs contributeurs à ces équipes successives.

De plus, le statut de direction et de rédaction de revue - et a fortiori de revue de transfert scientifique, toujours en délicat équilibre - n'est pas des plus prisés, en raison sans doute du gabarit et de la diversité des travaux obscurs qui y sont rattachés: recherche de financement, évaluation scientifique, corrections, quant ce n'est réécriture, édition, pour n'en citer que quelques-uns. Sont ainsi posées les conditions de travail qui ne facilitent guère l'implication, si l'on pense que pour la direction, il y a une seule décharge d'enseignement par année, attribuée par financement externe (la personne directrice absorbe trois cours plutôt que quatre, sans compter les directions de recherche et sa propre production scientifique). À ce chapitre, nous subissons durement les interminables compressions budgétaires de notre institution. De facto, la direction et la rédaction d'une revue sont déqualifiées.

Mais il y a plus: à la différence des autres revues universitaires, Frontières ne relève pas d'une association disciplinaire ou interuniversitaire, d'un département, d'un groupe de recherche qui dépasse un minuscule noyau. En d'autres termes, elle ne peut compter sur une logique structurelle qui contribue à garantir la contribution effective et continue de professeurs. Cette fragilité statutaire a pu constituer une force relative pour notre revue, dans la mesure où nous avons pu en revanche être appuyés et stimulés par des personnes bénévoles provenant de divers milieux, particulièrement depuis 1997. C'est beaucoup grâce à elles que la campagne de financement a obtenu sa résonance sociale, en 1998. Mais aujourd'hui, on ne se presse pas aux portes pour animer le travail d'un tel groupe (en dépit de l'intérim assuré pour quelques mois), de sorte que cette fragilité nous oblige à faire le point douloureusement.

L'existence même de la mort pose le principe de réalité. Traduisons le tout de go pour nous: les études sur la mort, du moins la revue qui en a émané en 1988, ne serait pas la priorité réelle d'un assez grand nombre de professeurs pour que la vitalisation de ce véhicule de savoirs qu'est la revue soit institutionnellement assurée: de la sorte, Frontières ne publiera plus sous l'égide de l'UQAM à compter de l'été 2001 (volume 13), à la suite de quoi la revue devra trouver une autre lancée...

Cet état de fait est d'autant plus troublant que Frontières reste la seule revue interdisciplinaire dans son domaine et que l'arborescence des situations et des questions autour de la mort se révèle de plus en plus pertinente pour nos manières de vivre individuelles et collectives. De la sorte, la revue a pu gagner une place comme référence chez beaucoup d'intervenants ainsi que dans la population. Frontières reçoit aussi de plus en plus de témoignages d'estime. On lui propose des textes et on lui refuse très rarement une invitation à collaborer - comme auteur... Elle bénéficie d'un budget équilibré, sous l'oeil compétent de Diane Laflamme. Au quotidien, elle existe des soins attentifs d'une responsable de production chevronnée. Alors?

Quoiqu'il advienne, survie ou dans le temps, autre vie, que soient vivement et chaleureusement remerciés les lectrices et les lecteurs, abonnés ou de passage, ainsi que les collaborateurs, pour leur soutien, leurs suggestions, et pour toutes les manifestations de confiance en l'esprit de Frontières, pluriel plus que dogmatique, interrogateur plus qu'affirmatif, plus décapant que confortant, bref, cherchant une ampleur qui a corps et justesse.

Luce Des Aulniers

Directrice sortante

La publication de Frontières, vol. 12, no 1, automne 1999, intitulée "Suicides, générations et culture " a bénéficié de l'appui

du ministère de la Santé et des Services sociaux du Québec. 